\title{
Interacción suelo-zapata corrida
}

\author{
A. Deméneghi-Colina y H. Sanginés-García \\ Departamento de Geotecnia, Facultad de Ingeniería, UNAM \\ E-mail:agusdc@lycos.com
}

(recibido: junio de 2001; aceptado: agosto de 2001)

\begin{abstract}
Resumen
Se presenta un procedimiento para el análisis de la interacción suelo-estructura a base de un marco con barras ortogonales, apoyado sobre una zapata corrida. Se muestra la forma de tomar en cuenta la rigidez del terreno de cimentación para realizar el análisis de la estructura. El procedimiento se ilustra con un ejemplo sencillo resuelto paso a paso.
\end{abstract}

Descriptores: interacción suelo-estructura, rigidez del suelo y estructura, cálculo de deformaciones en suelo.

\section{Abstract}

This paper presents a procedure for the analysis of the soil-structure interaction based on a frame with orthogonal barsleaning on a common foundation. It is shown how to take into consideration the rigidity of the soil to analyze the structure. The procedure is ilustrated with a simple example which is solved step by step.

Keywords: soil-structure interaction, soil and structure rigidity, structural analysis, compute of soil deformations.

\section{Introducción}

La interacción suelo-estructura es aquella parte de la ingeniería que estudia las deformaciones del terreno de cimentación cuando éstas se ven afectadas por la presencia y rigidez de la propia estructura. La influencia de la estructura puede ser en condiciones estáticas, lo cual es tratado por la interacción estática suelo-estructura, o puede ser en condiciones dinámicas, que cae en el campo de la interacción dinámica suelo-estructura.

Se conocen como métodos de interacción estática suelo-estructura aquellos procedimientos que para el cálculo de las deformaciones del terreno de cimentación toman en cuenta la rigidez de la estructura. Todos estos métodos están basados en el principio de que en el contacto cimiento-terreno los desplazamientos tanto de la subestructura, como los del terreno son iguales, es decir, existe compatibilidad de deformaciones entre estructura y suelo.

En términos generales, el procedimiento de cálculo para la interacción suelo-estructura consiste en tres pasos: (a) se calculan los desplazamientos de la subestructura, (b) se calculan los desplazamientos del terreno de cimentación, y (c) se establece la compatibilidad de deformaciones entre estructura y suelo.

Podemos distinguir dos clases de situaciones en relación con la interacción: (i) cuando los cimientos están suficientemente separados, de tal forma que la carga sobre un apoyo no ejerce influencia sobre los desplazamientos de los apoyos vecinos (este fenómeno se presenta usualmente en zapatas aisladas), y (ii) cuando se trata de un cimiento continuo donde el desplazamiento de un punto de dicho cimiento está afectado por la carga repartida en toda la subestructura (es el caso de zapatas corridas o losas de cimentación).

En este artículo presentamos un procedimiento sencillo para llevar a cabo la interacción estática suelo-estructura a base de un marco con barras ortogonales entre sí, apoyado sobre una zapata corrida.

\section{Interacción suelo-cimiento continuo}

Sea un cimiento totalmente flexible con carga uniforme apoyado en un suelo cohesivo totalmente saturado. E1 asentamiento a largo plazo toma la forma indicada en la figura 1a (Sowers, 1962); el diagrama de reacción del terreno en este caso es igual al de la carga, es decir, la reacción es uniforme. Si dicho cimiento se apoya sobre un suelo 
friccionante, el asentamiento se distribuye como se indica en la figura $1 \mathrm{~b}$; por ser el cimiento totalmente flexible, la reacción del suelo es también uniforme.
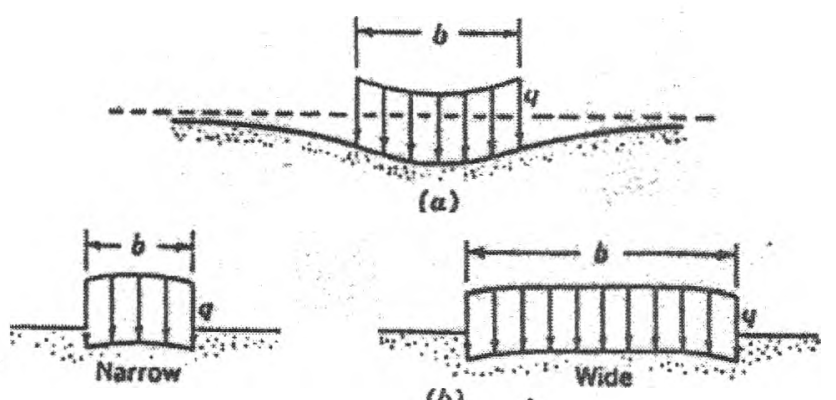

(b)

Diagrama de asentamiento de una carga uniforme sobre la superficie de una masa de suelo. (a) Material clástico, homogéneo e isótropo, tal como una arcilla saturada; (b) Material elástico y homogéneo, cuya rigidez aumenta con el confinamiento, tal como arena o grava. (Sowers, 1962)

\section{Figura 1}

Sea ahora, una placa de una rigidez infinita apoyada en una arcilla totalmente saturada (Figura 2a). El hundimiento es uniforme, pero el diagrama de reacción a largo plazo toma la forma indicada en la figura 2 a (Sowers, 1962). Si la placa se apoya sobre un suelo friccionante, el diagrama de reacción toma la forma de la figura $2 b$.

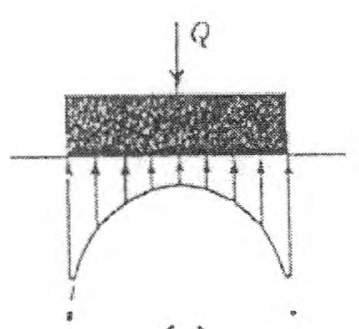

(a)

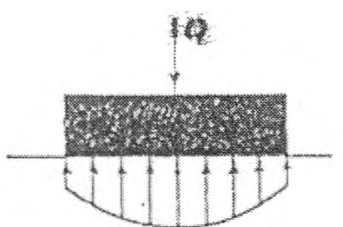

(b)
Presión de contacto en la base de una cimentación rígida. (a) Material elástico, homogéneo e isótropo, tal como una arcilla saturada; (b) Material elástico y homogéneo cuya rigidez aumenta con el confinamiento, tal como arena o grava. (Sowers, 1962)

\section{Figura 2}

Vemos entonces que los diagramas de asentamientos y de reacciones del terreno dependen de la clase de suelo y de la rigidez de la estructura. Un cimiento real puede quedar entre los dos casos extremos señalados, pues su rigidez no necesariamente es nula o infinita. En los siguientes incisos veremos cómo se realiza la interacción suelo-estructura para estructuras con cimentación de rigidez finita. Con el propósito de ilustrar el procedimiento, consideraremos el caso sencillo de un marco a base de barras ortogonales, apoyado en un terreno de comportamiento lineal.

\section{Interacción suelo-zapata corrida}

Consideremos un marco estructural con una cimentación a base de una zapata corrida (Figura 3a), en el cual se tratan de obtener los diagramas de asentamientos y de reacciones del terreno de cimentación (Figura 3, b y c).
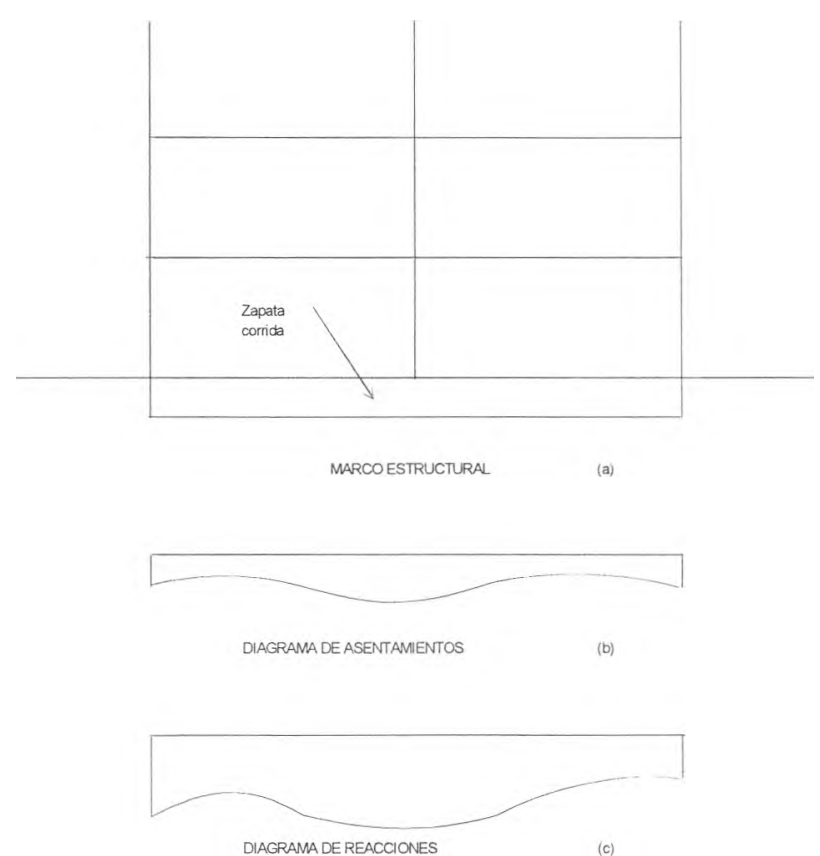

Figura 3. Marco estructural con cimentación a base de zapata corrida

Comencemos con el diagrama de reacciones. En el caso general, la forma del diagrama es diferente de una reacción uniforme (Figura 3c). Sustituyamos la curva de reacción del terreno por una serie de reacciones uniformes $r_{1}, r_{2}, \ldots$, $r_{n}$ (Figura 4a); el análisis estructural lo llevamos a cabo utilizando el método de rigideces, considerando las reacciones $r_{i}$ como incógnitas. A continuación, aplicando 
la tercera ley de Newton, adaptamos las cargas $r_{i}$ sobre el terreno (Figura 4b), y obtenemos los hundimientos de éste en función de las $r_{i}$, empleando el método de Chamecki (1956). El problema de la interacción se resuelve estableciendo la compatibilidad de deformaciones entre estructura y suelo, es decir, si el suelo está en contacto con la estructura de cimentación, las deformaciones de ambos medios deben ser iguales.

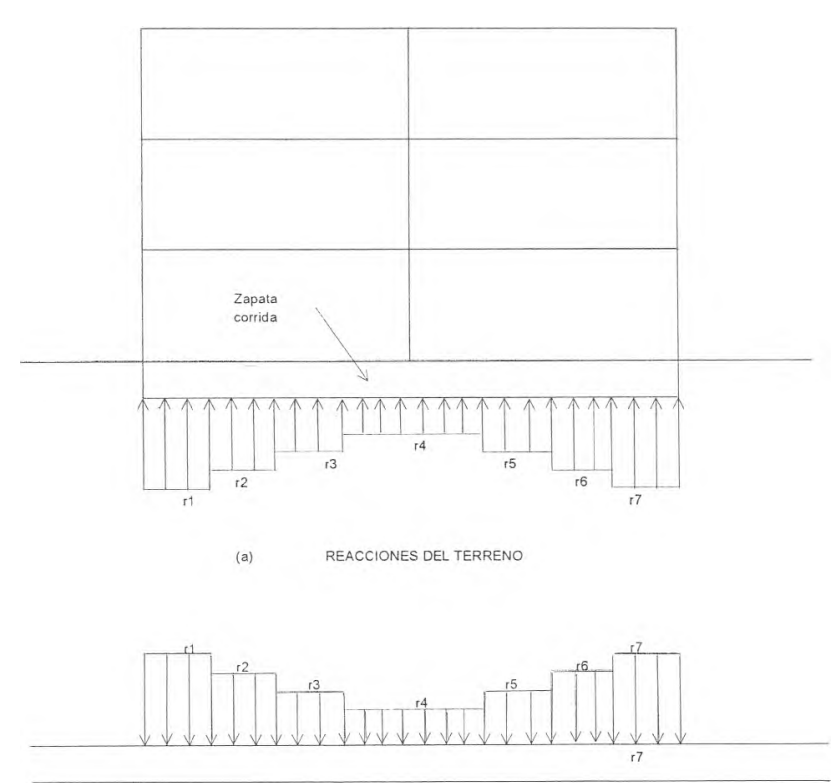

(b)

CARGAS SOBRE EL TERRENO

Figura 4. Cargas sobre la estructura y el suelo

\section{a) Análisis estructural}

El análisis estructural se realiza empleando el método de rigideces, en el cual, para que se satisfaga el equilibrio en los nudos de la estructura, se debe cumplir que

$$
\underline{K \delta}+\underline{P^{e}}+\underline{P_{c}}=0
$$

donde

$\underline{\mathrm{K}} \quad=$ matriz de rigidez de la estructura

$\underline{\delta}=$ vector de desplazamientos

$\underline{\mathrm{P}^{c}}=$ vector de cargas de empotramiento

$\underline{P_{c}}=$ vector de cargas concentradas
Las matrices de rigidez y los vectores de cargas de empotramiento se hallan con los valores del anexo 1 (marcos planos con barras ortogonales, sin considerar el acortamiento de barras).

En una barra de cimentación (Figura 5), el vector de cargas de empotramiento para una barra horizontal vale (véase el anexo 1).

$$
\underline{P}_{m}^{e}=\left[\begin{array}{ll}
\mathrm{wL}^{2} / 12-(11 / 192) \mathrm{L}^{2} \mathrm{r}_{r}-(5 / 192) \mathrm{L}^{2} \mathrm{r}_{\mathrm{s}} & \theta_{\theta_{p}} \\
-\mathrm{wL}^{2} / 12+(5 / 192) \mathrm{L}^{2} \mathrm{r}_{r}+(11 / 192) \mathrm{L}^{2} \mathrm{r}_{\mathrm{s}} & \theta_{q} \\
-\mathrm{wL} / 2+(13 / 32) \mathrm{Lr}_{\mathrm{r}}+(3 / 32) \mathrm{Lr}_{\mathrm{s}} & \delta_{r} \\
-\mathrm{wL} / 2+(3 / 32) \mathrm{Lr}_{\mathrm{r}}+(13 / 32) \mathrm{Lr}_{\mathrm{s}} & \delta_{s}
\end{array}\right.
$$

Para una barra vertical (anexo 1)

$$
\underline{P}_{m}^{e}=\left[\begin{array}{cc}
\mathrm{wL}^{2} / 12-(11 / 192) \mathrm{L}^{2} \mathrm{r}_{r}-(5 / 192) \mathrm{L}^{2} \mathrm{r}_{s} & \theta_{p} \\
-\mathrm{wL}^{2} / 12+(5 / 192) \mathrm{L}^{2} \mathrm{r}_{r}+(11 / 192) \mathrm{L}^{2} \mathrm{r}_{s} & \theta_{\theta_{q}} \\
\mathrm{wL} / 2+(13 / 32) \mathrm{Lr}_{\mathrm{r}}+(3 / 32) \mathrm{Lr}_{\mathrm{s}} & \delta_{u} \\
\mathrm{wL} / 2+(3 / 32) \mathrm{Lr}_{\mathrm{r}}+(13 / 32) \mathrm{Lr}_{\mathrm{s}} & \delta_{v}
\end{array}\right.
$$

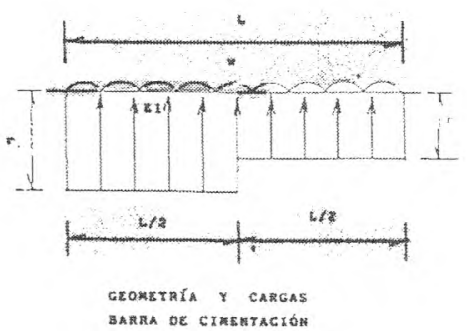

Figura 5. Geometría y cargas. Barra de cimentación (Deméneghi, 1996)

b) Cálculo de deformaciones del suelo

Las cargas que transmite la estructura al terreno de cimentación son iguales en magnitud y de sentido contrario a las reacciones del suelo sobre la estructura, por la tercera ley de Newton (Deméneghi, 1996). Calculemos los asentamientos del terreno en función de estas cargas: consideremos una reacción $\mathrm{r}_{\mathrm{k}}$ actuando en la superficie (Figura 6); la presión vertical vale $\mathrm{r}_{k} \mathrm{~d}_{\mathrm{k}} / \mathrm{a}_{\mathrm{k}}$, donde $\mathrm{d}_{\mathrm{k}} \mathrm{y} \mathrm{a}_{\mathrm{k}}$ son la longitud y el área en las que actúa la carga, respectivamente. En los siguientes párrafos consideraremos un suelo de comportamiento lineal.

El esfuerzo normal vertical en el punto $i j$, debido a la carga $r_{k}$, está dado por

$$
\sigma_{z i \mathrm{ijk}}=\mathrm{I}_{z i \mathrm{ijk}} \mathrm{r}_{\mathrm{k}} \mathrm{d}_{\mathrm{k}} / \mathrm{a}_{\mathrm{k}}
$$

donde $I_{z i j k}$ es el esfuerzo normal vertical producido por una presión unitaria actuando en el área k (Zeevaert, 1973). El esfuerzo normal vertical debido a todas las cargas $r_{k}$ vale 


$$
\sigma_{z i j}=\sum_{k=1}^{n r} I_{z i j k} r_{k} d_{k} / a_{k}
$$

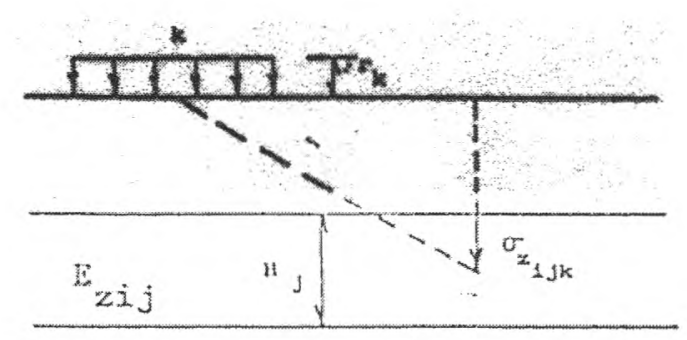

Figura 6. Cálculo de los hundimientos del terreno (Deméneghi, 1996)

En forma análoga

$$
\begin{aligned}
& \sigma_{x i j}=\sum_{k=1}^{n r} I_{x i j k} r_{k} d_{k} / a_{k} \\
& \sigma_{y i j}=\sum_{k=1}^{n r} I_{y i j k} r_{k} d_{k} / a_{k}
\end{aligned}
$$

Por otra parte, la deformación bajo el punto $i$ de un estrato de suelo de espesor $\mathrm{H}_{\mathrm{j}}$ está dada por

$$
\delta_{i j}=\left(H_{j} / E_{i j}\right)\left[\sigma_{z i j}-v\left(\sigma_{x i j}+\sigma_{y i j}\right)\right]
$$

donde $E_{i j}$ es el módulo de deformación del suelo y $v$ su relación de Poisson.

Sustituyendo las ecuaciones 4 a 6 en la ecuación 7

$$
\delta_{i j}=\left(H_{j} / E_{i j}\right) \sum_{k=1}^{n r}\left[I_{z i j k}-v\left(I_{x i j k}+I_{y j j k}\right)\right] r_{k} d_{k / a_{k}}
$$

Sea

$$
\begin{gathered}
I_{i j k}=I_{z i j k}-v\left(I_{x i j k}+I_{y i j k}\right) \\
\delta_{i j}=\left(H_{j} / E_{i j}\right) \sum_{k=1}^{n \pi} I_{i j k} r_{k} d_{k} / a_{k}
\end{gathered}
$$

Tomando en cuenta todos los estratos de subsuelo, y una posible deformación previa $\delta_{o i}$, la deformación del punto $i$ es

$$
\delta_{i}=\delta_{o i}+\sum_{j=1}^{n e}\left(H_{j} / E_{i j}\right) \sum_{k=1}^{n r} I_{i j k} r_{k} d_{k} / a_{k}
$$

\section{c) Compatibilidad de deformaciones}

En esta etapa se establece la compatibilidad de deformaciones entre estructura y suelo de cimentación, lo que equivale a considerar que tanto los desplazamientos de la estructura como los del terreno son iguales, es decir, que el suelo no se despega de la estructura (Deméneghi, 1996).

\section{Elementos mecánicos}

Los elementos mecánicos se obtienen con las ecuaciones contenidas en el anexo 2. Véase el anexo 1 para identificar los símbolos utilizados.

Los diagramas de fuerza cortante y de momento flexionante en una barra de la cimentación (Figura 7) se obtienen con las siguientes expresiones

$$
\mathrm{x} \leq \mathrm{L} / 2: \mathrm{V}=-\mathrm{V}_{\mathrm{r}}+\left(\mathrm{r}_{\mathrm{r}}-\mathrm{w}\right) \mathrm{x}
$$

$$
\begin{gathered}
M=-M_{p}-V_{r} x-\left(r_{r}-w\right) x^{2} / 2 \\
x \geq L / 2: V=-V_{r}-w x+r_{r} L / 2+r_{s}(x-L / 2) \\
M=-V_{r}-w x^{2} / 2+\left(r_{r} L / 2\right)(x-L / 4)+\left(r_{s} / 2\right)(x-L / 2)^{2}
\end{gathered}
$$

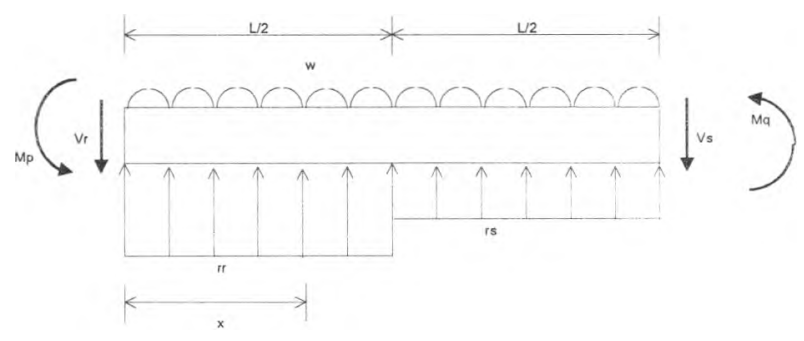

Figura 7. Elementos mecánicos (nudo sobre barra)

\section{Ejemplo}

Ilustremos el desarrollo del procedimiento de interacción con la zapata de la figura 8. El subsuelo está formado por dos estratos de arcilla totalmente saturada, con módulos de deformación no drenados $\mathrm{E}_{\mathrm{u}}$ de 500 y $560 \mathrm{t} / \mathrm{m}^{2}$, respectivamente. Se pide hacer el análisis de interacción suelo-estructura para condiciones a corto plazo, en condiciones no drenadas.

\section{a) Análisis estructural}

El análisis estructural se lleva a cabo empleando el método de rigideces. En la figura 9 y en la tabla 1 se muestran los 
grados de libertad; en la figura 10 se exhibe el sistema de cargas sobre la estructura. Las matrices de rigidez se obtienen con los valores del anexo 1 , dado que se trata de barras horizontales. Los vectores de cargas de empotramiento se calculan con la ecuación 2 .
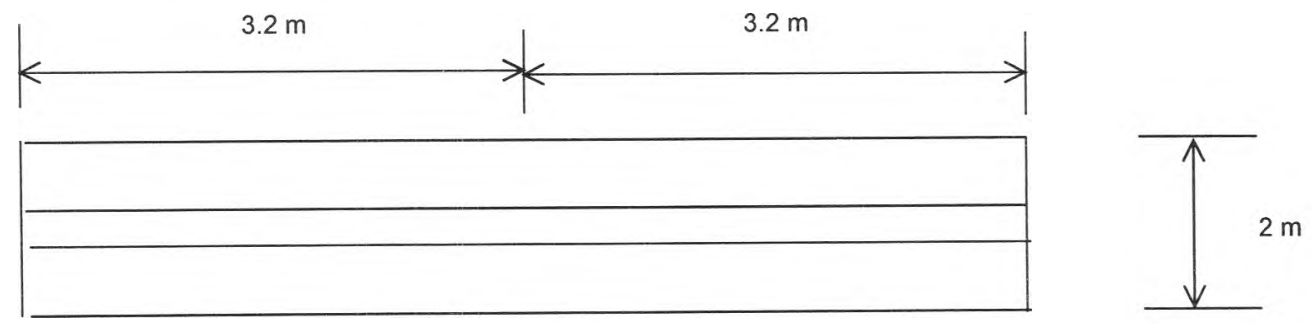

(a)

PLANTA

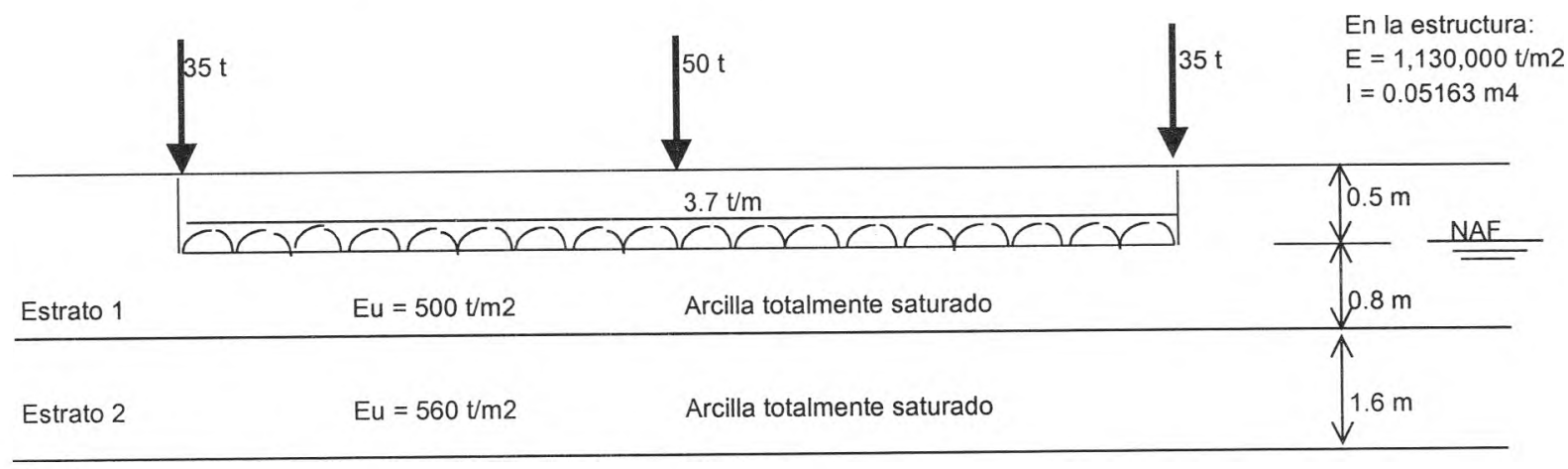

Roca

(b)

ELEVACIÓN

Figura 8. Características de estructura y terreno de cimentación
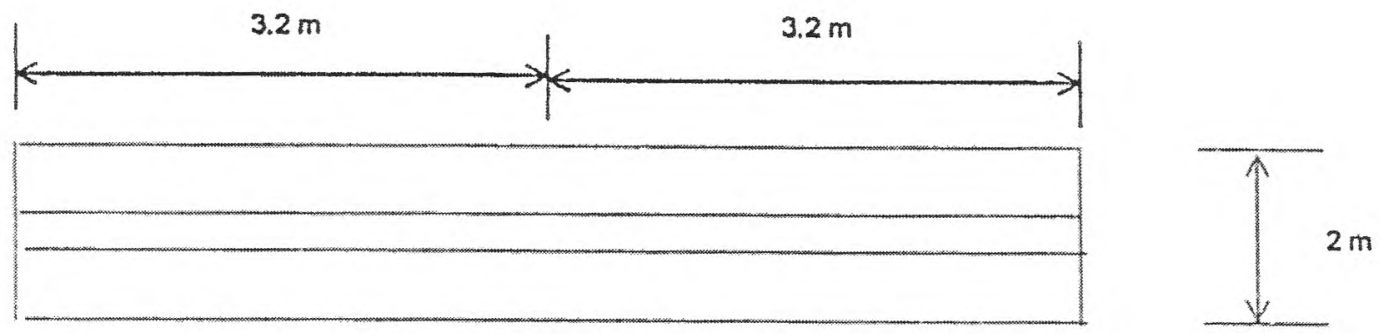

PLANTA

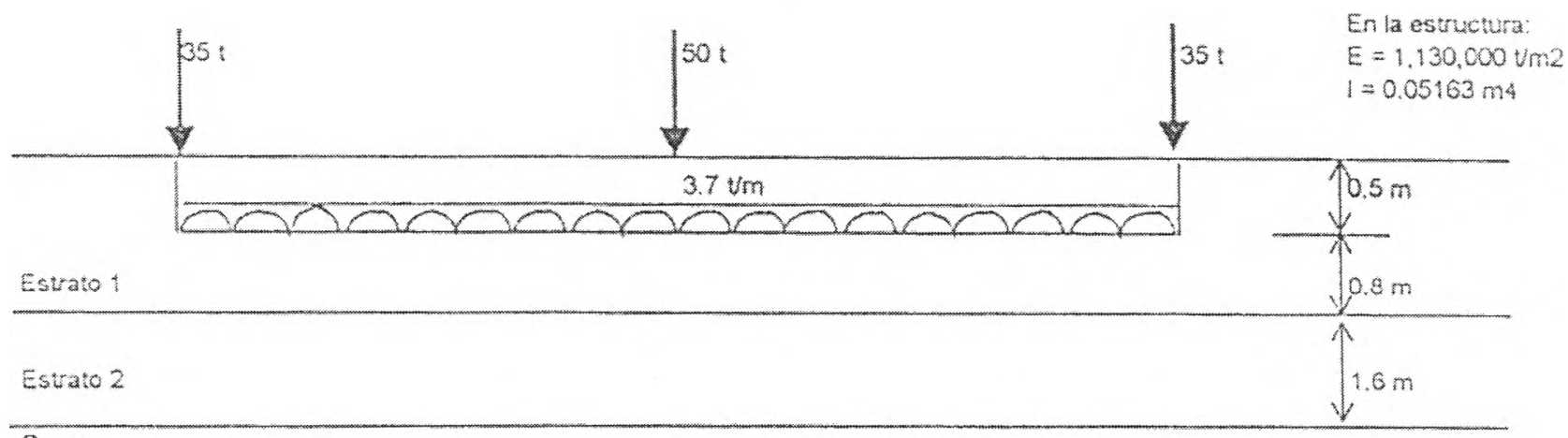




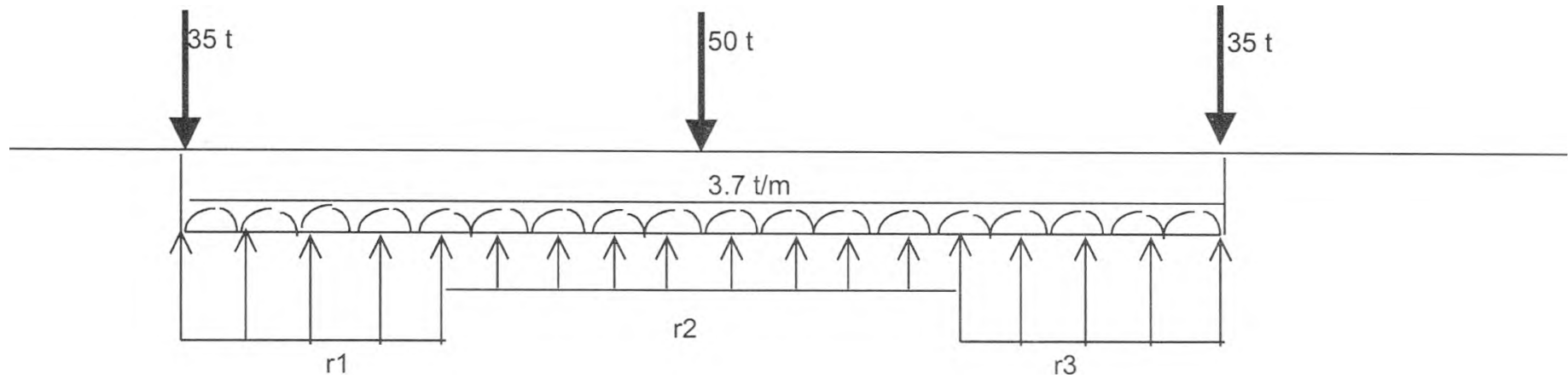

Figura 10. Sistema de cargas sobre la estructura

Matriz de rigidez. Barra 1

\begin{tabular}{ccccc}
\hline \hline$\theta_{4}$ & $\theta_{5}$ & $\delta_{1}$ & $\delta_{2}$ & \\
\hline 72927.375 & 36463.688 & -34184.707 & 34184.707 & $\theta_{4}$ \\
36463.688 & 72927.375 & -34184.707 & 34184.707 & $\theta_{5}$ \\
-34184.707 & -34184.707 & 21365.442 & -21365.442 & $\delta_{1}$ \\
34184.707 & 34184.707 & -21365.442 & 21365.442 & $\delta_{2}$ \\
\hline \hline
\end{tabular}

Matriz de rigidez. Barra 2

\begin{tabular}{ccccc}
\hline \hline$\theta_{5}$ & $\theta_{6}$ & $\delta_{2}$ & $\delta_{3}$ & \\
\hline 72927.375 & 36463.688 & -34184.707 & 34184.707 & $\theta_{5}$ \\
36463.688 & 72927.375 & -34184.707 & 34184.707 & $\theta_{6}$ \\
-34184.707 & -34184.707 & 21365.442 & -21365.442 & $\delta_{2}$ \\
34184.707 & 34184.707 & -21365.442 & 21365.442 & $\delta_{3}$ \\
\hline \hline
\end{tabular}

Tabla 1. Nuemeración de barras y grados de libertad

\begin{tabular}{ccccc}
\hline Barra & $\theta_{\mathrm{p}}$ & $\theta_{4}$ & $\delta_{\mathrm{r}}$ & $\delta_{\mathrm{s}}$ \\
1 & $\theta_{4}$ & $\theta_{5}$ & $\delta_{1}$ & $\delta_{2}$ \\
2 & $\theta_{5}$ & $\theta_{6}$ & $\delta_{2}$ & $\delta_{3}$ \\
\hline
\end{tabular}

Tabla 2. Matriz de rigidez de la estructura

\begin{tabular}{cccccl}
\hline Delta 1 & Delta 2 & Delta 3 & Theta 4 & Theta 6 & \\
\hline 21365.442 & -21365.442 & 0 & -34184.707 & 0 & Delta 1 \\
-21365.442 & 42730.884 & -21365.442 & 34184.707 & -34184.707 & Delta 2
\end{tabular}

\begin{tabular}{llllll}
-34184.707 & 34184.707 & 0 & 72927.375 & 0 & Theta 4 \\
\hline
\end{tabular}

Vector de cargas de empotramiento. Barra 1

$$
\underline{P}_{1}^{e}=\left[\left.\begin{array}{ll}
3.15733-0.58667 \mathrm{r}_{1}-0.26667 \mathrm{r}_{2} & 7 \theta_{4} \\
-3.15733+0.26667 \mathrm{r}_{1}+0.58667 \mathrm{r}_{2} & \mid \begin{array}{l}
\theta_{5} \\
-5.92+1.3 \mathrm{r}_{1}+0.3 \mathrm{r}_{2} \\
-5.92+0.3 \mathrm{r}_{1}+1.3 \mathrm{r}_{2}
\end{array}
\end{array}\right|_{\delta_{2}} ^{\delta_{1}}\right.
$$

Vector de cargas de empotramiento. Barra 2

$$
\underline{P}_{2}^{e}=\left[\begin{array}{ll}
3.15733-0.58667 \mathrm{r}_{2}-0.26667 \mathrm{r}_{3} & 7 \theta_{5} \\
-3.15733+0.26667 \mathrm{r}_{2}+0.58667 \mathrm{r}_{3} & \theta_{\theta_{6}} \\
-5.92+1.3 \mathrm{r}_{2}+0.3 \mathrm{r}_{3} & \delta_{2} \\
-5.92+0.3 \mathrm{r}_{2}+1.3 \mathrm{r}_{3} & \delta_{3}
\end{array} \delta_{3}\right.
$$

La matriz de rigidez de toda la estructura (tabla 2) es la suma de las matrices de rigidez de cada una de las barras. El vector de cargas de empotramiento de toda la estructura es la suma de los vectores de carga de empotramiento de cada una de las barras, el cual vale

$$
\underline{P}^{e}=\left[\begin{array}{ll}
-5.92+1.3 \mathrm{r}_{1}+0.3 \mathrm{r}_{2} & 7 \delta_{1} \\
-11.84+0.6 \mathrm{r}_{1}+2.6 \mathrm{r}_{2} & \left.\mid \begin{array}{l}
\delta_{2} \\
3.15733-0.58667 \mathrm{r}_{1}-0.26667 \mathrm{r}_{2}
\end{array}\right] \theta_{4}
\end{array}\right.
$$


(Sólo se muestran los renglones correspondientes a $\delta_{1}$, $\delta_{2}$ y $\theta_{4}$ porque, por simetría $\delta_{3}=\delta_{1}, \theta_{6}=-\theta_{4}$ y $\theta_{5}=0$ ).

El vector de cargas concentradas vale

$$
\underline{P}^{c}=\left[\begin{array}{r}
-35\rceil \delta_{1} \\
-50 \mid \delta_{2} \\
-35 \mid \delta_{3} \\
0 \mid \theta_{4} \\
0
\end{array}\right] \theta_{6}
$$

La condición de equilibrio de cargas en los nudos de la estructura conduce a la siguiente expresión (ecuación 1)

$$
\underline{K} \underline{\delta}+\underline{P}^{e}+\underline{P}^{c}=0
$$

Sustituyendo valores

$$
\left(\delta_{1}\right) \text { : }
$$

$$
\begin{gathered}
21365.442 \delta_{1}-21365.442 \delta_{2}-34184.707 \theta_{4} \\
+1.3 r_{1}+0.3 r_{2}-5.92-35=0
\end{gathered}
$$

$$
\left(\delta_{2}\right):
$$

$$
\begin{gathered}
-42730.884 \delta_{1}+42730.884 \delta_{2}+68369.414 \theta_{4} \\
+0.6 \mathrm{r}_{1}+2.6 \mathrm{r}_{2}-11.84-50=0 \\
\left(\theta_{4}\right): \\
-34184.707 \delta_{1}+34184.707 \delta_{2}+72927.375 \theta_{4} \\
-0.58667 \mathrm{r}_{1}-0.26667 \mathrm{r}_{2}+3.15733=0
\end{gathered}
$$

b) Cálculo de asentamientos

En el suelo, desarrollamos la ecuación 9 para $\mathrm{i}=1$ :

$$
\begin{gathered}
\delta_{1}=\left(\mathrm{H}_{1} / \mathrm{E}_{11}\right)\left(\mathrm{I}_{111} \mathrm{r}_{1} \mathrm{~d}_{1} / \mathrm{a}_{1}\right. \\
\left.+\mathrm{I}_{112} \mathrm{r}_{2} \mathrm{~d}_{2} / \mathrm{a}_{2}+\mathrm{I}_{113} \mathrm{r}_{3} \mathrm{~d}_{3} / \mathrm{a}_{3}\right)+\left(\mathrm{H}_{12} / \mathrm{E}_{12}\right)\left(\mathrm{I}_{121} \mathrm{r}_{1} \mathrm{~d}_{1} / \mathrm{a}_{1}\right. \\
\left.+\mathrm{I}_{122} \mathrm{r}_{2} \mathrm{~d}_{2} / \mathrm{a}_{2}+\mathrm{I}_{123} \mathrm{r}_{3} \mathrm{~d}_{3} / \mathrm{a}_{3}\right)
\end{gathered}
$$

Determinemos como ejemplo el valor de influencia $I_{111}$ $=I_{z 111}-v\left(I_{x 111}+I_{y 111}\right)$. Se coloca una presión unitaria $q=1$ $\mathrm{t} / \mathrm{m}^{2}$ en el área $\mathrm{a}_{1}$ (Figura 11) y se computan los esfuerzos normales $\sigma_{z}, \sigma_{x}$ y $\sigma_{y}$ debidos a esta carga, a la mitad del estrato 1 . Obtenemos

$$
\begin{aligned}
& \sigma_{z}=I_{z 111}=0.4868711 \mathrm{t} / \mathrm{m}^{2} \\
& \sigma_{x}=I_{x 111}=0.3181542 \mathrm{t} / \mathrm{m}^{2}
\end{aligned}
$$

$$
\sigma_{\mathrm{y}}=\mathrm{I}_{\mathrm{y} 111}=0.265932 \mathrm{t} / \mathrm{m}^{2}
$$$$
I_{111}=0.4868711-0.5(0.3181542+0.265932)=0.194828
$$

Los demás valores de influencia se determinan en forma similar. En la tabla 3 se presentan sus magnitudes.

Sustituyendo valores en la ecuación A

$$
\begin{aligned}
& \delta_{1}=(0.8 / 500)\left[(0.194828 / 2) r_{1}-(0.02614844 / 2) r_{2}\right. \\
& \left.-(0.00174077 / 2) r_{3}\right]+(1.6) /(560)\left[(0.23528931 / 2) r_{1}\right. \\
& \left.-(0.00780255 / 2) r_{2}-(0.00481864 / 2) r_{3}\right]
\end{aligned}
$$

Tomando en cuenta que $\mathrm{r}_{1}=\mathrm{r}_{3}$
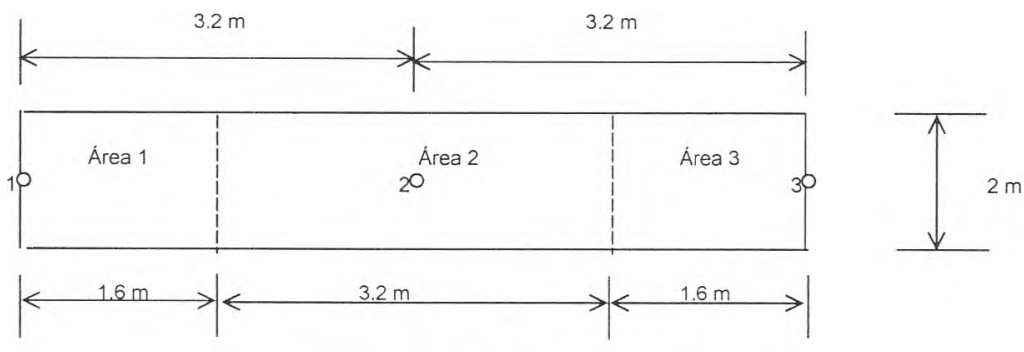

PLANTA

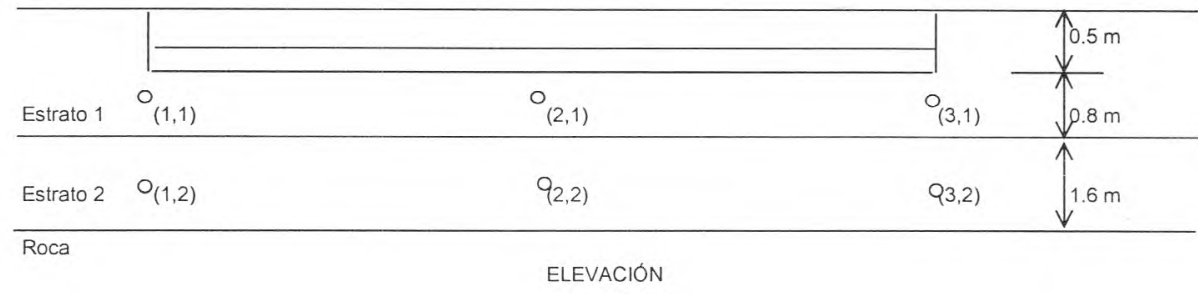

Figura 11. Cálculo de los valores de influencia 
Tabla 3. Valores de influencia. Relación de Poisson $=0.5$

\begin{tabular}{cccccc}
\hline Punto & Izijk & Ixijk & Iyijk & nu & lijk \\
\hline $1,1,1$ & 0.4868711 & 0.3181542 & 0.265932 & 0.5 & 0.194828 \\
$1,1,2$ & 0.00174314 & 0.05265242 & 0.00313073 & 0.5 & -0.02614844 \\
$1,1,3$ & $1.8865 \mathrm{E}-05$ & 0.00348082 & $3.8445 \mathrm{E}-05$ & 0.5 & -0.00174077 \\
$1,2,1$ & 0.2791369 & 0.05794332 & 0.02975186 & 0.5 & 0.23528931 \\
$1,2,2$ & 0.0402185 & 0.09123936 & 0.00480275 & 0.5 & -0.00780255 \\
$1,2,3$ & 0.000992 & 0.0114948 & 0.00012647 & 0.5 & -0.00481864 \\
$2,1,1$ & 0.00163603 & 0.04312015 & 0.00291786 & 0.5 & -0.02138298 \\
$2,1,2$ & 0.9737421 & 0.6363085 & 0.531864 & 0.5 & 0.38965585 \\
$2,1,3$ & 0.001636 & 0.04312015 & 0.00291786 & 0.5 & -0.021383 \\
$2,2,1$ & 0.03557754 & 0.06498982 & 0.00422196 & 0.5 & 0.00097165 \\
$2,2,2$ & 0.5582739 & 0.1158866 & 0.05950371 & 0.5 & 0.47057875 \\
$2,2,3$ & 0.03557754 & 0.06498982 & 0.00422196 & 0.5 & 0.00097165 \\
$3,1,1$ & $1.8865 \mathrm{E}-05$ & 0.00348082 & $3.8445 \mathrm{E}-05$ & 0.5 & -0.00174077 \\
$3,1,2$ & 0.00174314 & 0.05265242 & 0.00313073 & 0.5 & -0.02614844 \\
$3,1,3$ & 0.4868711 & 0.3181542 & 0.265932 & 0.5 & 0.194828 \\
$3,2,1$ & 0.000992 & 0.0114948 & 0.00012647 & 0.5 & -0.00481864 \\
$3,2,2$ & 0.0402185 & 0.09123936 & 0.00480275 & 0.5 & -0.00780255 \\
$3,2,3$ & 0.2791369 & 0.05794332 & 0.02975186 & 0.5 & 0.23528931 \\
\hline
\end{tabular}

$\delta_{1}=0.000483712 r_{1}-0.00003206525 r_{2}$

En forma análoga se obtiene

$\delta_{2}=-0.000031436 r_{1}+0.00098398 r_{2}$

c) Compatibilidad de deformaciones

La compatibilidad de deformaciones se cumple al resolver el sistema de ecuaciones 14 a 18 :

$\delta_{1}=0.014285 \mathrm{~m}, \delta_{2}=0.013224 \mathrm{~m}$

$\theta_{4}=0.00075212$

$r_{1}=30.487 \mathrm{t} / \mathrm{m}, r_{2}=14.413 \mathrm{t} / \mathrm{m}$

[Nota: Es importante que los módulos de deformación $\mathrm{E}_{\mathrm{ij}}$ se determinen considerando el efecto de la presión de confinamiento en el terreno, el hecho de que la curva esfuerzo-deformación unitaria de los suelos es no lineal, así como la posible variación con el tiempo de las propiedades mecánicas (Sanginés, 2000)].

Los elementos mecánicos los obtenemos con las expresiones del anexo 2. Como ejemplo, el momento en el grado de libertad $5\left(\theta_{5}\right)$ de la barra 1 se calcula con la siguiente expresión

$\mathrm{M}_{\mathrm{q}}=-\mathrm{wL}^{2} / 12+(5 / 192) \mathrm{L}^{2} \mathrm{r}_{\mathrm{r}}+(11 / 192) \mathrm{L}^{2} \mathrm{r}_{\mathrm{s}}+(2 \mathrm{EI} / \mathrm{L})$ $\theta_{\mathrm{p}}+(4 \mathrm{EI} / \mathrm{L}) \theta_{\mathrm{q}}-\left(6 \mathrm{EI} / \mathrm{L}^{2}\right) \delta_{\mathrm{r}}+\left(6 \mathrm{EI} / \mathrm{L}^{2}\right) \delta_{\mathrm{s}}$

$\mathrm{M}_{5}=-3.7(3.2)^{2} / 12+(5 / 192)(3.2)^{2}(30.487)+(11 / 192)$

$(3.2)^{2}(14.413)+(36463.688)(0.00075212)+(72927.375)$

$(0)-(34184.707)(0.014285)+(34184.707)(0.013224)$

$=4.583 \mathrm{t} . \mathrm{m}$.

Los demás elementos mecánicos se hallan en forma similar. En la tabla 4 se muestran sus magnitudes.Los diagramas de fuerza cortante y momento flexionante se hallan utilizando las ecuaciones 10 a 13. En la figura 12 se exhiben los diagramas de asentamiento, de reacciones, de fuerza cortante y de momento flexionante.

Tabla 4. Elementos mecánicos en los nudos de la estructura

\begin{tabular}{ccccc}
\hline Barra & $\mathrm{M}_{\mathrm{p}}$ & $\mathrm{M}_{\mathrm{q}}$ & $\mathrm{V}_{\mathrm{r}}$ & $\mathrm{V}_{\mathrm{s}}$ \\
\hline & $\mathrm{t} . \mathrm{m}$ & $\mathrm{t} . \mathrm{m}$ & $\mathrm{t}$ & $\mathrm{t}$ \\
1 & 0 & 4.583 & 35 & 25 \\
2 & -4.583 & 0 & 25 & 35 \\
\hline \hline
\end{tabular}




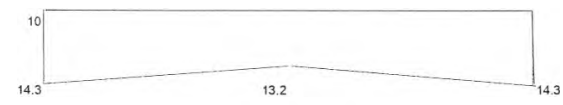

ASENTAMIENTO, cm

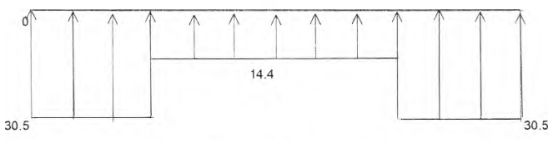

REACCIONES DEL TERRENO. UTm

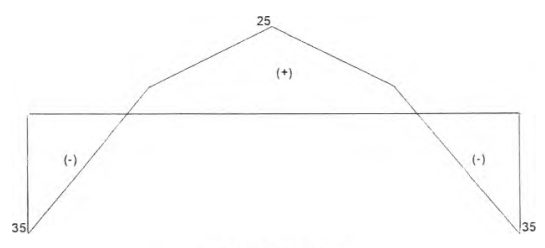

FUERZA CORTANTE,

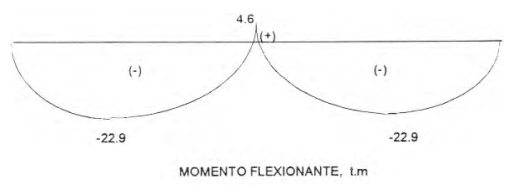

Figura 12. Diagramas de asentamiento, reacciones, fuerza cortante y movimiento flexionante

\section{Anexo 1}

Matriz de rigidez y vector de cargas de empotramiento

Matriz de rigidez barra horizontal

\begin{tabular}{|c|c|c|c|c|}
\hline Theta $\mathrm{p}$ & Theta q & Delta r & Delta s & \\
\hline $4 \mathrm{EI} / \mathrm{L}$ & $2 \mathrm{EI} / \mathrm{L}$ & $-6 \mathrm{EI} / \mathrm{L}^{2}$ & $6 \mathrm{EI} / \mathrm{L}^{2}$ & Theta $\mathrm{p}$ \\
\hline $2 \mathrm{EI} / \mathrm{L}$ & $4 \mathrm{EI} / \mathrm{L}$ & $-6 \mathrm{EI} / \mathrm{L}^{2}$ & $6 \mathrm{EI} / \mathrm{L}^{2}$ & Theta \\
\hline$-6 \mathrm{EI} / \mathrm{L}^{2}$ & $-6 \mathrm{EI} / \mathrm{L}^{2}$ & $12 \mathrm{EI} / \mathrm{L}^{3}$ & $-12 \mathrm{EI} / \mathrm{L}^{3}$ & Delta \\
\hline $6 \mathrm{EI} / \mathrm{L}^{2}$ & $6 \mathrm{EI} / \mathrm{L}^{2}$ & $-12 \mathrm{EI} / \mathrm{L}^{3}$ & $12 \mathrm{EI} / \mathrm{L}^{3}$ & Delta s \\
\hline \multicolumn{5}{|c|}{ Vector de cargas de empotramiento barra horizontal } \\
\hline $\mathrm{wL}^{2} / 12$ & Theta $\mathrm{p}$ & & & \\
\hline$-w L^{2} / 12$ & Theta $\mathrm{q}$ & & & \\
\hline$-w L / 2$ & Delta r & & & \\
\hline$-w L / 2$ & Delta s & & & \\
\hline \multicolumn{5}{|c|}{ Matriz de rigidez barra vertical } \\
\hline Theta $p$ & Theta q & Delta u & Delta v & \\
\hline $4 \mathrm{EI} / \mathrm{L}$ & $2 \mathrm{EI} / \mathrm{L}$ & $6 \mathrm{EI} / \mathrm{L}^{2}$ & $-6 \mathrm{EI} / \mathrm{L}^{2}$ & Theta $p$ \\
\hline $2 \mathrm{EI} / \mathrm{L}$ & $4 \mathrm{EI} / \mathrm{L}$ & $6 \mathrm{EI} / \mathrm{L}^{2}$ & $-6 \mathrm{EI} / \mathrm{L}^{2}$ & Theta \\
\hline $6 \mathrm{EI} / \mathrm{L}^{2}$ & $6 \mathrm{EI} / \mathrm{L}^{2}$ & $12 \mathrm{EI} / \mathrm{L}^{3}$ & $-12 \mathrm{EI} / \mathrm{L}^{3}$ & Delt \\
\hline$-6 \mathrm{EI} / \mathrm{L}^{2}$ & $-6 \mathrm{EI} / \mathrm{L}^{2}$ & $-12 \mathrm{EI} / \mathrm{L}^{3}$ & $12 \mathrm{EI} / \mathrm{L}^{3}$ & Delta \\
\hline \multicolumn{5}{|c|}{ Vector de cargas de empotramiento barra vertical } \\
\hline$w L^{2} / 12$ & Theta $\mathrm{p}$ & & & \\
\hline$-w L^{2} / 12$ & Theta q & & & \\
\hline$w L / 2$ & Delta u & & & \\
\hline $\mathrm{wL} / 2$ & Delta v & & & \\
\hline
\end{tabular}
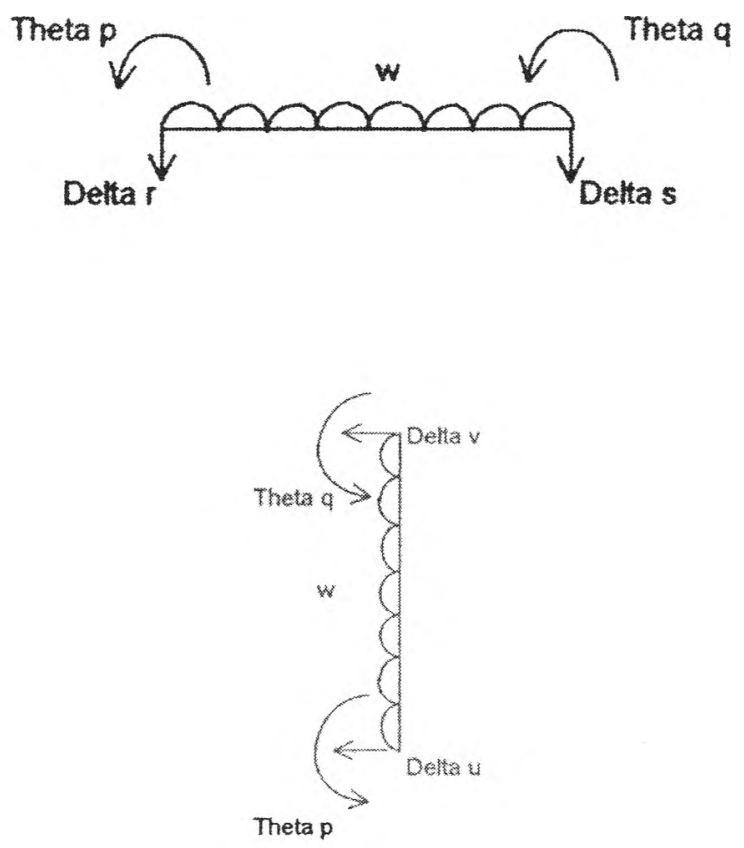


\section{Anexo 2}

Cálculo de elementos mecánicos

Barra horizontal

$\mathrm{M}_{\mathrm{p}}=\mathrm{wL}^{2} / 12-(11 / 192) \mathrm{L}^{2} \mathrm{r}_{\mathrm{r}}-(5 / 192) \mathrm{L}^{2} \mathrm{r}_{\mathrm{s}}+(4 \mathrm{EI} / \mathrm{L}) \theta_{\mathrm{p}}+(2 \mathrm{EI} / \mathrm{L}) \theta_{\mathrm{q}}-\left(6 \mathrm{EI} / \mathrm{L}^{2}\right) \delta_{\mathrm{r}}+\left(6 \mathrm{EI} / \mathrm{L}^{2}\right) \delta_{\mathrm{s}}$

$M_{q}=-w^{2} / 12+(5 / 192) L^{2} r_{r}+(11 / 192) L^{2} r_{s}+(2 E I / L) \theta_{p}+(4 E I / L) \theta_{q}-\left(6 E I / L^{2}\right) \delta_{r}+\left(6 E I / L^{2}\right) \delta_{s}$

$V_{r}=-w L / 2+(13 / 32) L_{r_{r}}+(3 / 32) L_{r_{s}}-\left(6 E I / L^{2}\right) \theta_{p}-\left(6 E I / L^{2}\right) \theta_{q}+\left(12 \mathrm{EI} / \mathrm{L}^{3}\right) \delta_{r}-\left(12 \mathrm{EI} / \mathrm{L}^{3}\right) \delta_{s}$

$\mathrm{V}_{\mathrm{s}}=-\mathrm{wL} / 2+(3 / 32) \mathrm{Lr}_{\mathrm{r}}+(13 / 32) \mathrm{Lr}_{\mathrm{s}}+\left(6 \mathrm{EI}^{2} \mathrm{~L}^{2}\right) \theta_{\mathrm{p}}+\left(6 \mathrm{EI} / \mathrm{L}^{2}\right) \theta_{\mathrm{q}}-\left(12 \mathrm{EI} / \mathrm{L}^{3}\right) \delta_{\mathrm{r}}+\left(12 \mathrm{EI} / \mathrm{L}^{3}\right) \delta_{\mathrm{s}}$

Nota: En las barras de la superestructura tomar $r_{r}=r_{s}=0$ ]

Barra vertical

$\mathrm{M}_{\mathrm{p}}=\mathrm{wL}^{2} / 12+(4 \mathrm{EI} / \mathrm{L}) \theta_{\mathrm{p}}+(2 \mathrm{EI} / \mathrm{L}) \theta_{\mathrm{q}}+\left(6 \mathrm{EI} / \mathrm{L}^{2}\right) \delta_{\mathrm{u}}-\left(6 \mathrm{EI} / \mathrm{L}^{2}\right) \delta_{\mathrm{v}}$

$\mathrm{M}_{\mathrm{q}}=-\mathrm{wL}^{2} / 12+(2 \mathrm{EI} / \mathrm{L}) \theta_{\mathrm{p}}+(4 \mathrm{EI} / \mathrm{L}) \theta_{\mathrm{q}}+\left(6 \mathrm{EI} / \mathrm{L}^{2}\right) \delta_{\mathrm{u}}-\left(6 \mathrm{EI} / \mathrm{L}^{2}\right) \delta_{\mathrm{v}}$

$\mathrm{V}_{\mathrm{u}}=\mathrm{wL} / 2-\left(6 \mathrm{EI} / \mathrm{L}^{2}\right) \theta_{\mathrm{p}}-\left(6 \mathrm{EI} / \mathrm{L}^{2}\right) \theta_{\mathrm{q}}-\left(12 \mathrm{EI} / \mathrm{L}^{3}\right) \delta_{\mathrm{u}}+\left(12 \mathrm{EI} / \mathrm{L}^{3}\right) \delta_{v}$

\section{Conclusiones}

Se presentó una forma sencilla de llevar a cabo el análisis de interacción suelo-estructura con base en un marco cimentado sobre una zapata corrida. El resultado de este análisis permite obtener los diagramas de asentamiento, de reacciones, de fuerza cortante y de momento flexionante.En la práctica, conviene elaborar programas de computadora que realicen el análisis de interacción con el procedimiento presentado en los incisos anteriores. También es aconsejable utilizar para fines prácticos un mayor número de barras y de estratos del subsuelo (una barra de cimentación se puede dividir para fines de análisis en dos o más barras de menor tamaño, y un estrato de suelo se puede dividir en dos o más estratos de menor espesor).

\section{Referencias}

Chamecki S. (1956). Structural Rigidity in Calculating Settlements. Jour Soil Mech Found Div, Proc ASCE, Vol 88, N SM1.

Deméneghi A. (1996). Interacción estática suelo-estructura, considerando efectos de torsión y acortamiento de barras. XVIII Reunión Nacional de Mecánica de Suelos, Vol. 1: 303-310, Morelia, Soc. Mex. Mec. Suelos.

Sanginés H. (2000). Interacción suelo-estructura en suelos friccionantes. Tesis de maestría en División de Estudios de Posgrado, Facultad de Ingeniería, UNAM.

Sowers G.F. (1962). Shallow Foundations, Leonards G.A., McGraw-Hill.

Zeevaert L. (1973). Foundation Engineering for Difficult Subsoil Conditions, Van Nostrand Reinhold. institución. Ha sido profesor de mecánica del medio continuo, mecánica de suelos y cimentaciones desde 1975. Realizó investigaciones sobre cálculo de deformaciones en los suelos e interacción suelo-estructura.

Héctor Sanginés-García. Es ingeniero civil y maestro en ingeniería por la Facultad de Ingeniería UNAM, ha impartido diversas asignaturas como mecánica del medio continuo, mecánica de suelos y cimentaciones desde 1981. Ha realizado investigaciones acerca de cálculo de deformaciones en los suelos e interacción suelo-estructura. 